Pacific Journal of Mathematics

ON THE CONJUGATING REPRESENTATION OF A FINITE 


\title{
ON THE CONJUGATING REPRESENTATION OF A FINITE GROUP
}

\author{
RICHARD L. ROTH
}

\begin{abstract}
A natural permutation representation for any finite group is the conjugating representation $T$ : for each $g \in G$, $T(g)$ is the permutation on the set $\{x \mid x \in G\}$ given by $T(g)(x)=g x g^{-1}$. Frame, Solomon and Gamba have studied some of its properties. This paper considers the question of which complex irreducible representations occur as components of $T$, in particular the conjecture that any such representation whose kernel contains the center of $G$ is a component of $T$. This conjecture is verified for a few special cases and a number of related results are obtained, especially with respect to the one-dimensional components of $T$.
\end{abstract}

In $\S 2$ we see that the conjecture does hold for groups of "central type" which were studied by DeMeyer and Janusz in [4]. In $\S 3$ we obtain further information with respect to the linear characters of $G$; it is shown that if $G / H$ is a cyclic group then the number of irreducible characters of $G$ which are induced from irreducible characters of $H$ is the same as the number of conjugacy classes of $G$ having the property that the centralizers of their elements belong to $H$. This number is precisely the multiplicity in the conjugating representation of a linear character of $G$ whose kernel is $H$.

Notation. $G$ is a finite group with conjugacy classes $C_{1}, C_{2}, \cdots$, $C_{k}$. $\quad \chi^{1}, \chi^{2}, \cdots, \chi^{k}$ are the irreducible complex characters of $G$. $\left\{g_{1}, g_{2} \cdots, g_{k}\right\}$ will be a set of representatives of the conjugacy classes with $g_{j} \in C_{j}$ for $j=1,2, \cdots, k$. We let $T$ denote the conjugating representation of $G$ defined above and $\theta$ will be the character of $G$ corresponding to $T$. The transitivity classes (orbits) under $T$ are then $C_{1}, \cdots, C_{k}$ and restricting $T$ to the set $C_{i}$ gives the corresponding transitive permutation representation $T^{i}$ where $i=1,2, \cdots, k$. Let $\varphi^{i}$ be the character of $T^{i}$ for each $i$, so that $\theta=\sum_{i=1}^{k} \varphi^{i}$.

If $\eta$ and $\lambda$ are two complex-valued characters on $G$, then $(\eta, \lambda)$ will denote the usual "inner product" given by

$$
(\eta, \lambda)=|G|^{-1} \sum_{g \in G} \eta(g) \lambda \overline{(g)}
$$

where $\lambda \overline{(g)}$ is the complex conjugate of $\lambda(g)$, and $|G|$ is the order of $G$. $Z$ will denote the center of the group $G$. The kernel of $\lambda$, denoted $\operatorname{Ker} \lambda$, is to mean the kernel of a representation affording the 
character $\lambda$. The underlying field is always assumed to be the complex numbers. If $g \in G$ then $C(g)$ denotes the centralizer of $g$ in $G$. For general background material the reader is referred to [3] and [5].

1. General properties of the conjugating representation.

Lemma 1.1. $\theta=\sum_{i=1}^{k} a_{i} \chi^{i}$ where $a_{i}=\sum_{j=1}^{k} \chi^{i}\left(g_{j}\right)$.

Proof. This was proved by Solomon in [11]. See also Theorem 6.5 in [5]. This lemma is also noted without proof in [7, p. 192].

We use lemma 1.1 to give a new proof of the following theorem due to Frame (see [6]).

THEOREM 1.2.

$$
\theta=\sum_{i=1}^{k} \chi^{i} \overline{\chi^{i}}
$$

Proof.

$$
\text { Let } \sum_{i=1}^{k} \chi^{i} \bar{\chi}^{i}=\sum_{j=1}^{k} b_{j} \chi^{j}
$$

Then

$$
\begin{aligned}
& b_{j}=\left(\sum_{i=1}^{k} \chi^{i} \overline{\chi^{i}}, \chi^{j}\right)=\sum_{i=1}^{k}\left(\chi^{i} \overline{\chi^{i}}, \chi^{j}\right) \\
& =\sum_{i=1}^{k} \frac{1}{|G|} \sum_{g \in G}^{k} \chi^{i}(g) \chi^{\bar{i}(g)} \chi^{\bar{j}(g)} \\
& =\sum_{g \in G}^{k} \frac{1}{|G|}\left(\sum_{i=1}^{k} \chi^{i}(g) \chi^{\bar{i}(g)}\right) \overline{\chi^{j}}(g) \\
& =\sum_{g \in G} \frac{1}{|G|} \frac{|G|}{h(g)} \chi^{\bar{j}(g)}=\sum_{l=1}^{k} \overline{\chi^{j}}\left(g_{l}\right) \\
& =\overline{\sum_{l=1}^{k} \chi^{j}\left(g_{l}\right)}=\bar{a}_{j}=a_{j} \quad(\text { by }(1.1)) \text {. }
\end{aligned}
$$

(Here $h(g)$ denotes the number of elements in the conjugacy class of $g$ ). So

$$
\theta=\sum_{j=1}^{k} a_{j} \chi^{j}=\sum_{j=1}^{k} b_{j} \chi^{j}=\sum_{i=1}^{k} \chi^{i} \overline{\chi^{i}}
$$

Lemma 1.3. (Frame... [6]). If $\chi^{j}$ appears in the decomposition of $\theta$ (i.e., if $a_{j}>0$ ) then $Z$ is contained in $\operatorname{Ker} \chi^{j}$.

Proof. If $z \in Z$, then $T(z)$ corresponds to the identical transfor- 
mation and $z$ must be in the kernel of each of the irreducible components of $T$.

We conjecture that the converse is also true, i.e.,

CONJECTURE 1.4. If $\chi^{j}$ is a complex irreducible character and $Z \subseteq \operatorname{Ker} \chi^{j}$ then $\alpha_{j}>0$.

In seeking to prove this conjecture it is of interest to examine the more specific problem of finding conditions on $C_{j}$ and $\chi^{i}$ such that $\chi^{i}$ appears in the decomposition of $\varphi^{j}$, i.e., in the special conjugating representation afforded by the $j^{\text {th }}$ conjugacy class. To that end we have

Lemma 1.5. Let $\chi$ be a complex irreducible character of $G$.

(i) $\chi$ occurs in the decomposition of $\phi^{j}$ precisely $m$ times where $m$ is the multiplicity of the 1-representation of the restriction of $\chi$ to $C\left(g_{j}\right)$.

(ii) If $\chi$ is a linear character, then $\chi$ occurs in the decomposition of $\phi^{j}$ at most once and occurs once precisely if $C\left(g_{j}\right) \subseteq \operatorname{Ker} \chi$.

REMARK. The above lemma is independent of the choices of representatives $g_{j}$ for the conjugacy classes.

Proof. Under the transitive permutation representation $T^{j}$ of $G$ defined on the set $C_{j}, C\left(g_{j}\right)$ is the subgroup of $G$ of elements which leave the given element $g_{j}$ fixed. $T^{j}$ may thus be regarded as the representation induced from the 1-representation on $C\left(g_{j}\right)$. By the Frobenius reciprocity theorem,

$$
m=\left(\chi, \varphi^{j}\right)_{G}=\left(\chi \mid C\left(g_{j}\right), 1\right)_{C\left(g_{j}\right)}
$$

where 1 here stands for the 1-character of $C\left(g_{j}\right)$.

(ii) By ( i ) if $\chi$ is linear it occurs as a component of $\varphi^{j}$ precisely if $\chi$ restricted to $C\left(g_{j}\right)$ is the 1-representation in which case $m=1$ and $\operatorname{Ker} \chi \supseteqq C\left(g_{j}\right)$.

2. Groups of central type. In the paper of DeMeyer and Janusz ([4]), a group of central type is defined to be a group having an irreducible character $\chi$ on $G$ with $\chi(1)^{2}=[G: Z]$. We see in the following theorem that conjecture (1.4) holds for these groups.

THEOREM 2.1. Let $G$ be a finite group of central type. Then every irreducible character $\psi$ with $Z \subseteq \operatorname{Ker} \psi$ appears as a component of the conjugating character $\theta$ at least $n$ times where $n$ is the degree of $\psi$. 
Proof. Let $\chi$ be an irreducible character of $G$ with $\chi(1)^{2}=[G: Z]$. By Corollary 1 in [4], $\chi(g)=0$ for $g \notin Z$. Let $\psi$ be an irreducible character of degree $n$ with $Z \leqq \operatorname{Ker} \psi$. For $g \in Z, \psi(g) \chi(g)=n \chi(g)$. For $g \notin Z, \psi(g) \chi(g)=0=n 0=n \chi(g)$. I.e., $\psi \chi=n \chi$.

Now by $[5,(6.6)]$ or by $[3$, p. 274], we have

$$
(\psi, \chi \bar{\chi})=(\chi, \psi \chi)=(\chi, n \chi)=n \text {. }
$$

By Theorem 1.2, $\theta=\sum_{i=1}^{k} \chi^{i} \overline{\chi^{i}}$ so $\psi$ appears in $\theta$ at least $n$ times.

REMARK. The above proof can be adapted to prove the converse of Corollary 1 in [4], namely that if $\chi(g)=0$ for $g \notin Z$ then $\chi(1)^{2}=[G: Z]$. Professor Janusz has noted to the author that this follows more directly by observing that $1=(\chi, \chi)=(1 /|G|) \chi(1)^{2}|Z|$.

As an example of these groups we consider the following:

THEOREM 2.2. Let $G$ be a nilpotent group of class 2 with cyclic center. Then $G$ is of central type and every irreducible character $\psi$ with $Z \subseteq \operatorname{Ker} \psi$ appears as a component of $\theta$.

Proof. Kochendörffer has shown in [9] that a nilpotent group with cyclic center has a faithful irreducible complex character $\chi$ (i.e., see Theorem 4). By Lemma 9, p. 1482 in [8], $\chi(g)=0$ for $g \notin Z$. By the remark preceding the theorem we see that $G$ is of central type, and the second statement follows from (2.1) (or its proof).

REMARK. We note that in the above case $G^{\prime} \leqq Z$, so if $Z \leqq \operatorname{Ker} \psi$, $\psi$ is of necessity a linear character. In the next section we concentrate on the relation of linear characters to the conjugating representation.

\section{Linear characters.}

THEOREM 3.1. Let $\lambda$ be a linear character of $G$ and $\rho$ an irreducible character of $G$. Then $\lambda \rho=\rho \Leftrightarrow \rho$ is induced from an irreducible representation on $\operatorname{Ker} \lambda$.

Proof. Suppose $\rho$ is induced from an irreducible character of the normal subgroup $\operatorname{Ker} \lambda$. Then $\rho(x)=0$ for $x \notin \operatorname{Ker} \lambda$ and since $\lambda(x)=1$ for $x \in \operatorname{Ker} \lambda$, we have $\lambda \rho=\rho$.

Conversely, suppose that $\lambda \rho=\rho$. Let $R$ be a representation of $G$ on a complex vector space $V$ such that $R$ affords the character $\rho$. Then there exists an invertible linear transformation $S$ of $V$ such that $S R(g) S^{-1}=\lambda(g) R(g)$ for all $g$ in $G$. Thus $S R(g)=\lambda(g) R(g) S$ for all $g$ in $G$. 
Now let $v$ be an eigenvector of $S$ and $\mu$ be the corresponding eigenvalue.

$$
S R(g) v=\lambda(g) R(g) S v=\lambda(g) R(g) \mu v=(\lambda(g) \mu) R(g) v .
$$

Hence $R(g) v$ is an eigenvector of $S$ with eigenvalue $\lambda(g) \mu$. Thus each distinct value of $\lambda$ gives a distinct eigenvalue of $S$. Let $h_{1}=1, \cdots, h_{r}$ be coset representatives for a coset decomposition of $G$ modulo the kernel of $\lambda$. Then $\lambda\left(h_{1}\right), \lambda\left(h_{2}\right) \cdots \lambda\left(h_{r}\right)$ are precisely the distinct values that $\lambda$ takes on. For $i=1,2, \cdots r$ let $V_{i}$ be the eigenspace of $V$ consisting of all eigenvectors of $S$ with eigenvalue $\lambda\left(h_{i}\right) \mu$. If $g \in G$ and $v_{i} \in V_{i}$, then $R(g) v_{i}$ is an eigenvector with value $\lambda(g) \lambda\left(h_{i}\right) \mu=$ $\lambda\left(h_{j}\right) \mu$ for some $j$, by equation (1). $R(g)$ thus maps $V_{i}$ injectively into $V_{j}$. $R\left(g^{-1}\right)$ similarly maps $V_{j}$ injectively into $V_{i}$ so both subspaces have the same dimension and $R(g)$ maps $V_{i}$ injectively onto $V_{j}$. The subspace $V_{1} \oplus V_{2}+\cdots \oplus V_{r}$ is evidently invariant under the representation $R$ and since $R$ is irreducible, $V=V_{1} \oplus \cdots \oplus V_{r}$. Also, $\left\{V_{1}, \cdots, V_{r}\right\}$ forms a system of imprimitivity for $V . R(g)$ leaves $V_{1}$ invariant precisely if $g \in \operatorname{Ker} \lambda$. Hence by Theorem 50.2 in [3], $V_{1}$ affords a representation of $\operatorname{Ker} \lambda$ which induces the representation $R$ of $G$. Clearly this representation of $\operatorname{Ker} \lambda$ must be irreducible since otherwise $R$ would not be irreducible.

Let $H$ be any normal subgroup of a group $G$ and $\psi_{1}$ and $\psi_{2}$ characters of $H$. If there exists $g \in G$ such that $\psi_{1}(x)=\psi_{2}\left(g x g^{-1}\right)$ for all $x \in H$, then we say that $\psi_{1}$ and $\psi_{2}$ are $G$-conjugate (see [3, p. 278, Ex. 6; also p. 343]). The irreducible characters of $H$ are thus divided up into "G-conjugacy classes". Let $N(\psi)=$ the "normalizer" of $\dot{\psi}$ in $\left\{G=g \in G \mid \psi(x)=\psi\left(g x g^{-1}\right)\right.$ all $\left.x \in G\right\}$.

THEOREM 3.2. Let $G$ be a finite group, $H$ a normal subgroup such that $G / H$ is cyclic. The following four numbers are then equal:

$a=$ the number of conjugacy classes $C_{i}$ such that $C\left(g_{i}\right) \subseteq H$.

$b=$ the number of $G$-conjugacy classes of irreducible characters $\psi$ of $H$ such that $N(\psi) \subseteq H$.

$c=$ the multiplicity of a linear character $\lambda$ in $\theta$, where $\lambda$ is any linear character with $\operatorname{Ker} \lambda=H$.

$d=$ the number of distinct irreducible characters of $G$ which are induced from irreducible characters of $H$.

Proof. Let $\lambda$ be any linear character of $G$ with $H=\operatorname{Ker} \lambda$; since $G / H$ is cyclic there exist linear characters satisfying this condition. By (1.5), part (ii), $\lambda$ occurs in the conjugating representation as many times as there are conjugacy classes $C_{j}$ with $C\left(g_{j}\right) \subseteq \operatorname{Ker} \lambda=H$. Thus $a=c$. By Theorem 1.2, $\theta=\sum \chi^{i} \bar{\chi}^{i}$. Now $\left(\lambda, \chi^{i} \bar{\chi}^{i}\right)=\left(\chi^{i}, \lambda \chi^{i}\right)=1$ 
or 0 depending on whether $\chi^{i}=\lambda \chi^{i}$ or not. Hence if $\lambda$ occurs in $\theta c$ times then there are precisely $c$ irreducible characters $\chi^{i}$ of $G$ such that $\lambda \chi^{i}=\chi^{i}$. By Theorem 3.1 these are precisely the characters of $G$ induced from irreducible characters of $H$, so $c=d$.

Let $\psi$ be an irreducible character of $H$. Then $\psi^{G}$ is irreducible precisely if $\psi^{g} \neq \psi$ for $g \notin H$ (by (45.5) in [3]). This means that $\psi^{g}=\psi$ implies that $g \in H$; i.e., $N(\psi) \subseteq H$. By Exercise 5, p. 278, in [3] two conjugate characters induce the same character of $G$. Now (45.6) in [3] applied in the case that $H_{1}=H_{2}$ shows that two nonconjugate irreducible characters can't induce the same irreducible character of $G$. Hence $b=d$.

We describe the constant from (3.2) in still another way. $G / H$ may be considered as a group operating by conjugation on the set of conjugacy classes $D_{1}, \cdots, D_{t}$ of $H$. Let $\left\{D_{1}, \cdots, D_{m}\right\}$ be an orbjt under this operation; i.e., $\bigcup_{i=1}^{m} D_{i}$ is a conjugacy class of $G$ contained in $H$. In the following lemma, the phrase " $G / H$ operates regularly on the orbit $\left\{D_{1}, \cdots, D_{m}\right\}$ " means $G / H$ permutes the set transitively and no element except the identity leaves any element fixed. The following lemma shows that $a$ of (3.2) equals the number of orbits on which $G / H$ acts regularly.

Lemma 3.3. $G / H$ operates regularly on the orbit $\left\{D_{1}, \cdots, D_{m}\right\}$ if and only if $C(x) \subseteq H$ where $x \in \bigcup_{i=1}^{m} D_{i}$.

(Note this is independent of the choice of $x_{\text {. }}$ )

Proof. Say $G / H$ is regular on $\left\{D_{1}, \cdots, D_{m}\right\}$ and $g \in C(x)$. If $x \in D_{i}$ then $g H$ operating on the orbit $\left\{D_{1}, \cdots, D_{m}\right\}$ fixes $D_{i}$ so $g H=H$ and $g \in H$, i.e., $C(x) \subseteq H$.

Conversely, say $C(x) \subseteq H$. Let $g \in G$ and suppose $g H$ fixes $D_{1}$ (for example). Let $x \in D_{1}, g x g^{-1}=x^{\prime}$ with $x^{\prime} \in D_{1}$. Then there exists $h \in H$ such that $h x h^{-1}=x^{\prime}$. Hence $h^{-1} g \in C(x) \subseteq H$ and so $g \in H$. Thus $g H=H$ and $G / H$ operates regularly on the orbit.

REMARK. Using a lemma of Brauer (Lemma 1, $\$ 6$ in [1]) an alternate proof can be given to show that $a=b$ in Theorem 3.2. For $G / H$ is cyclic and if $r=|G / H|$ then the number of orbits of length $r$ under the action of $G / H$ on the conjugacy classes of $H$ equals the number of orbits of length $r$ in the action of $G / H$ on the characters of $H$. The latter number is in fact $b$ while the former equals $a$ by Lemma 3.3. (See [10, Proposition 1.5] for a similar use of another lemma of Brauer).

We now verify that conjecture (1.4) holds in one more special case. 
THEOREM 3.4. Let $G$ be a finite group and $p$ a prime such that $p \mid G$ but $p^{2} \nmid G$. Let $\lambda$ be a linear character $G$ taking on exactly $p$ values. If $Z \subseteq \operatorname{Ker} \lambda$ then $\lambda$ occurs as a component of the conjugating character $\theta$.

Proof. By the Schur-Zassenhaus theorem ([3, (7.5)]) we may regard $G$ as the semidirect product of $\mathrm{Ker} \lambda$ and a cyclic group $P$ or order $p$. The elements $\neq 1$ of $P$ induce nontrivial automorphisms of Ker $\lambda$ by conjugation (since $Z \cong \operatorname{Ker} \lambda$ ). Theorem II, p. 89 of Burnside's book ([2]) states: "An isomorphism of a group $G$ whose order contains a prime factor which does not occur in the order of $G$ must interchange some of the conjugate sets of G". Thus if $\alpha$ is one of the nontrivial automorphisms of $P$, since it is of order $p$, there must be $p$ classes $D_{1}, \cdots, D_{p}$ of conjugacy classes of $\operatorname{Ker} \lambda$ regularly permuted in a cycle. By (3.3) and (3.2) the multiplicity of $\lambda$ in $\theta$ is at least 1 .

\section{BIBLIOGRAPHY}

1. R. Brauer, On the connection between the ordinary and modular characters of groups of finite order, Ann. of Math. 42 (1941), 926-935.

2. W. Burnside, Theory of Groups of Finite Order, second edition, Dover, New York, 1955.

3. C. W. Curtis and I. Reiner, Representation Theory of Finite Groups and Associative Algebras, Interscience, New York, 1962.

4. F. R. DeMeyer and Gerald J. Janusz, Finite groups with an irreducible representation of large degree, Math. Z. 108 (1969), 145-153.

5. Walter Feit, Character of Finite Groups, W. A. Benjamin, Inc., New YorkAmsterdam, 1967.

6. J. S. Frame, On the reduction of the conjugating representation of a finite group, Bull. Amer. Math. Soc. 53 (1948), 584-589.

7. R. Gamba, Representations and classes in groups of finite order, J. Math. Phys. 9 (1968), 186-192.

8. George Glauberman, Correspondence of characters for relatively prime operator groups, Canad, J. Math. 20 (1968), 1465-1488.

9. R. Kochendörffer, Über treue irreduzible Darstellungen endlicher Gruppen, Math. Nachrichrichten 1 (1948), 25-39.

10. Gary M. Seitz, M-groups and the supersolvable residual, Math. Z. 110 (1969), 101-122.

11. Louis, Solomon, On the sum of the elements in the character table of a finite, group, Proc. Amer. Math. Soc. 12 (1961), 962-963.

Received May 11, 1970.

UNIVERSITY OF COLORADO

Boulder, Colorado 



\section{PACIFIC JOURNAL OF MATHEMATICS}

\section{EDITORS}

H. SAMELson

Stanford University

Stanford, California 94305

R. R. PHelPS

University of Washington

Seattle, Washington 98105
J. Dugundu

Department of Mathematics

University of Southern Californle

Los Angeles, California 9.0007

RICHARD ARENS

University of California

Los Angeles, California 9.0024

\section{ASSOCIATE EDITORS}
E. F. BECKENBACH
B. H. NeumanN
F. WOLE
K. Yoshida

\section{SUPPORTING INSTITUTIONS}

UNIVERSITY OF BRITISH COLUMBIA

CALIFORNIA INSTITUTE OF TECHNOLOGY

UNIVERSITY OF CALIFORNIA

MONTANA STATE UNIVERSITY

UNIVERSITY OF NEVADA

NEW MEXICO STATE UNIVERSITY

OREGON STATE UNIVERSITY

UNIVERSITY OF OREGON

OSAKA UNIVERSITY

UNIVERSITY OF SOUTHERN CALIFORNIA

\author{
STANFORD UNIVERSITY \\ UNIVERSITY OF TOKYO \\ UNIVERSITY OF UTAH \\ WASHINGTON STATE UNIVERSITY \\ UNIVERSITY OF WASHINGTON \\ AMERICAN MATHEMATICAL SOCIETY \\ CHEVRON RESEARCH CORPORATION \\ NAVAL WEAPONS CENTER
}

The Supporting Institutions listed above contribute to the cost of publication of this Journal, but they are not owners or publishers and have no responsibility for its content or policies.

Mathematical papers intended for publication in the Pacific Journal of Mathematics should be in typed form or offset-reproduced, (not dittoed), double spaced with large margins. Underline Greek letters in red, German in green, and script in blue. The first paragraph or two must be capable of being used separately as a synopsis of the entire paper. The editorial "we" must not be used in the synopsis, and items of the bibliography should not be cited there unless absolutely necessary, in which case they must be identified by author and Journal, rather than by item number. Manuscripts, in duplicate if possible, may be sent to any one of the four editors. Please classify according to the scheme of Math. Rev. Index to Vol. 39. All other communications to the editors should be addressed to the managing editor, Richard Arens, University of California, Los Angeles, California, 90024.

50 reprints are provided free for each article; additional copies may be obtained at cost in multiples of 50 .

The Pacific Journal of Mathematics is published monthly. Effective with Volume 16 the price per volume (3 numbers) is $\$ 8.00$; single issues, $\$ 3.00$. Special price for current issues to individual faculty members of supporting institutions and to individual members of the American Mathematical Society: $\$ 4.00$ per volume; single issues $\$ 1.50$. Back numbers are available.

Subscriptions, orders for back numbers, and changes of address should be sent to Pacific Journal of Mathematics, 103 Highland Boulevard, Berkeley, California, 94708.

PUBLISHED BY PACIFIC JOURNAL OF MATHEMATICS, A NON-PROFIT CORPORATION

Printed at Kokusai Bunken Insatsusha (International Academic Printing Co., Ltd.), 7-17, Fujimi 2-chome, Chiyoda-ku, Tokyo, Japan. 


\section{Pacific Journal of Mathematics}

\section{Vol. 36, No. 2 December, 1971}

George E. Andrews, On a partition problem of H. L. Alder ............ 279

Thomas Craig Brown, An interesting combinatorial method in the theory of locally finite semigroups .......................... 285

Yuen-Kwok Chan, A constructive proof of Sard's theorem ............. 291

Charles Vernon Coffman, Spectral theory of monotone Hammerstein

operators...................................... 303

Edward Dewey Davis, Regular sequences and minimal bases .......... 323

Israel (Yitzchak) Nathan Herstein and Lance W. Small, Regular elements in

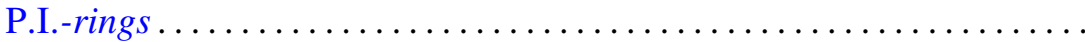

Marcel Herzog, Intersections of nilpotent Hall subgroups ..............

W. N. Hudson, Volterra transformations of the Wiener measure on the space

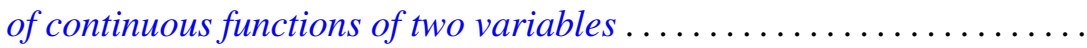

J. H. V. Hunt, An n-arc theorem for Peano spaces ................ 351

Arnold Joseph Insel, A decomposition theorem for topological group

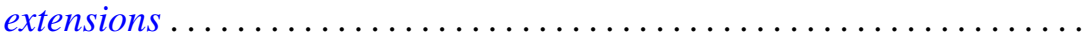

Caulton Lee Irwin, Inverting operators for singular boundary value

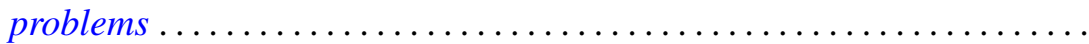

Abraham A. Klein, Matrix rings of finite degree of nilpotency ............ 387

Wei-Eihn Kuan, On the hyperplane section through a rational point of an algebraic variety...

John Hathway Lindsey, II, On a six-dimensional projective representation of $\mathrm{PSU}_{4}(3)$

Jorge Martinez, Approximation by archimedean lattice cones ...

J. F. McClendon, On stable fiber space obstructions .........

Mitsuru Nakai and Leo Sario, Behavior of Green lines at the Kuramochi boundary of a Riemann surface ....................

Donald Steven Passman, Linear identities in group rings. I. .

Donald Steven Passman, Linear identities in group rings. II ...

David S. Promislow, The Kakutani theorem for tensor products of

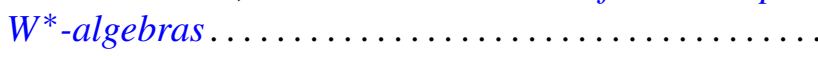

Richard Lewis Roth, On the conjugating representation of a finite group

Bert Alan Taylor, On weighted polynomial approximation of entire functions...

William Charles Waterhouse, Divisor classes in pseudo Galois

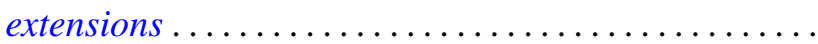

Chi Song Wong, Subadditive functions ...

Ta-Sun $\mathrm{Wu}$, A note on the minimality of certain bitransformation groups 